\title{
Multi-Stage Blind Clustering Equaliser
}

\author{
S. Chen, S. McLaughlin, P.M. Grant and B. Mulgrew
}

\begin{abstract}
A multi-stage blind clustering algorithm is proposed for equalisation of multi-level quadrature amplitute modulation ( $M$-QAM) channels. A hierarchical decomposition divides the task of equalising a high-order QAM channel into much simpler sub-tasks. Each sub-task can be accomplished fast and reliably using a blind clustering algorithm derived originally for $4-$ QAM signals. The constant modulus algorithm (CMA) is used as a benchmark to assess this multi-stage blind equaliser. It is demonstrated that the new blind algorithm achieves much faster convergence and is very robust when input symbols are not sufficiently white. This multi-stage clustering equaliser only requires slightly more computations than the CMA and, like the latter, its computational complexity does not increase as the levels of digital symbols increase.
\end{abstract}

\section{INTRODUCTION}

Blind equalisation techniques can be classified into three categories. The first class of blind adaptive schemes constructs a transversal equaliser using various Bussgang algorithms [1]-[3]. Most of the practical emphasis has been focused on the schemes known as Godard's algorithms. In particular, the CMA has a very simple computational requirement and is widely applied to high-order QAM signals [4],[5]. The second class of blind adaptive schemes identifies a channel model using techniques of higher-order cumulants [6]-[8], and then designs an equaliser based on the channel model obtained. This second class of blind equalisers, although very general and powerful, requires extensive computation. The third class of blind equalisers employs some joint channel and data estimation technique to approximate the maximum likelihood sequence estimation [9]- [11], which is computationally very expensive.

For 4-QAM signals, Karaoguz and Ardalan [12] derived a blind equaliser based on maximizing the a-posteriori (MAP) probability density function (p.d.f.) of the equaliser output subject to the equaliser weights, and demonstrated its superior performance over some of the well-known blind equalisers. Karaoguz and Ardalan's algorithm, referred to as the soft decision-directed blind algorithm in [12], is a Bussgang-type algorithm well fitted to the finite nature of digital symbol constellation. To avoid any confusion, this

Paper approved by Jack H. Winters, the Editor for Equalization of the IEEE Communications Society. Manuscript received January 6 , 1993; revised September 14, 1993 and February 7, 1994. This work was supported by the U.K. Science and Engineering Research Council under award GR/G/53095. The work of S. McLaughlin was supported by the U.K. Royal Society. This paper was presented in part at the IEEE International Conference on Communications, ICC'93, Geneva, Switzerland, May 23-26, 1993.

$\mathrm{S}$. Chen is with the Department of Electrical and Electronic Engineering, the University of Portsmouth, Anglesea Building Portsmouth PO1 3DJ, United Kingdom.

S. McLaughlin, P.M. Grant and B. Mulgrew are with the Department of Electrical Engineering, the University of Edinburgh, King's Buildings, Edinburgh EH9 $3 \mathrm{JL}$, United Kingdom.

IEEE Log Number 9410855 algorithm will be referred to as the blind clustering algorithm.

The present study extends the blind clustering algorithm to $M$-QAM $(M>4)$ signals. A straightforward application of this blind algorithm to $M-\mathrm{QAM}$ signals may not be a good idea and this is explained in simple geometric terms. Such a direct extension can further encounter the difficulty of increased complexity. By decomposing the task of reconstructing the data constellation into appropriate sub-tasks, a multi-stage blind clustering procedure is derived. Because each sub-task is very easy to achieve, this blind adaptive algorithm can accomplish fast convergence. Furthermore, its complexity does not increase as the levels of the data increase, and is always equal to that of the 4-QAM case. The performance of the multi-stage blind clustering equaliser is compared with that of the CMA using simulated channels.

\section{Blind Equalisation}

Consider the baseband model of a digital communication channel characterized by a finite impulse response (FIR) filter. The received signal is given by

$$
r(k)=\sum_{i=0}^{n-1} a_{i} s(k-i)+e(k),
$$

where $n$ is the length of the channel impulse response, $a_{i}=a_{i R}+j a_{i I}$ are the complex channel tap weights, the complex symbol sequence $s(k)=s_{R}(k)+j s_{I}(k)$ is assumed to be independently identically distributed (i.i.d.), $e(k)=e_{R}(k)+j e_{I}(k)$ is an i.i.d. complex Gaussian white noise with $\mathrm{E}\left[e_{R}^{2}(k)\right]=\mathrm{E}\left[e_{I}^{2}(k)\right]=\sigma_{e}^{2}$, and $\mathrm{E}[\cdot]$ denotes the expectation operator. The symbol constellation is $M$ QAM defined by

$$
s_{i l}=(2 i-Q-1)+j(2 l-Q-1), \quad 1 \leq i, l \leq Q,
$$

where $Q=\sqrt{M}=2^{L}$, and $L$ is an integer. The equaliser has a FIR structure defined as

$$
y(k)=\sum_{i=0}^{m-1} w_{i} r(k-i),
$$

where $m$ is the order of the equaliser and is assumed to be large enough, and $w_{i}=w_{i R}+j w_{i J}$ are the complex equaliser weights. The equaliser has a delay $d \approx m / 2$.

A family of blind adaptive algorithms called Godard's algorithms [2] adjusts the equaliser weight vector $\mathbf{w}=$ $\left[w_{0} \cdots w_{m-1}\right]^{T}$ by minimizing the cost function

$$
\bar{J}_{q}(\mathbf{w})=\mathrm{E}\left[\left(|y(k)|^{q}-\Delta_{q}\right)^{2}\right],
$$

using a gradient algorithm, where $q$ is a positive integer and $\Delta_{q}=\mathrm{E}\left[|s(k)|^{2 q}\right] / \mathrm{E}\left[|s(k)|^{q}\right]$. The case of $q=2$ is 0090-6778/95\$4.00 (C) 1995 IEEE 
known as the CMA [3], which is by far the most popular blind equaliser for high-order QAM constellations. It has a computational complexity comparable to that of the least mean square algorithm. The CMA is designed to penalize deviations of the equaliser output from a constant modulus. Although $M-\mathrm{QAM}$ symbols do not fall on the circle of radius $\sqrt{\Delta_{2}}$, it is known that $\bar{J}_{2}(\mathbf{w})$ is minimized at the weight solution which restores the signal constellation. Under certain conditions, the CMA converges to this solution subject to a possible phase shift. An important assumption to guarantee this convergence is that the input symbol sequence $s(k)$ is i.i.d.. In some practical situations, this condition is violated and false convergence of the CMA can occur. A scenario of this kind is described in [4].

\section{The BLIND Clustering ALgorithm}

When the equaliser weights have been correctly chosen, the equaliser output can be expressed as $y(k)=$ $s(k-d)+v(k)$, where $v(k)$ is approximately Gaussian white noise. Thus when the equalisation is accomplished, the equaliser output can be modelled approximately by $M$ Gaussian clusters with means

$$
y_{i l}=s_{i l}, \quad 1 \leq i, l \leq Q,
$$

and an approximate covariance

$$
\left[\begin{array}{cc}
\mathrm{E}\left[v_{R}^{2}(k)\right] & \mathrm{E}\left[v_{R}(k) v_{I}(k)\right] \\
\mathrm{E}\left[v_{I}(k) v_{R}(k)\right] & \mathrm{E}\left[v_{I}^{2}(k)\right]
\end{array}\right] \approx\left[\begin{array}{cc}
\rho & 0 \\
0 & \rho
\end{array}\right] .
$$

Denote the a-priori probability of $y_{q l}$ as $p_{q l}$. The aposteriori p.d.f. of $y(k)$ is

$$
\eta(\mathbf{w}, y(k))=\sum_{q=1}^{Q} \sum_{l=1}^{Q} \frac{p_{q l}}{2 \pi \rho} \exp \left(-\frac{\left|y(k)-y_{q l}\right|^{2}}{2 \rho}\right) .
$$

When the equaliser weights have not yet been set correctly, $y(k)$ does not satisfy the model (7) of $M$ Gaussian clusters. However, from (7), a bootstrap optimization process can be formed to adapt the equaliser by maximizing the criterion $\bar{\eta}(\mathbf{w})=\mathrm{E}[\eta(\mathbf{w}, y(k))]$. Because all the $p_{q l}, 1 \leq q, l \leq Q$, can be assumed to be equal, the criterion $\bar{\eta}(\mathbf{w})$ is equivalent to

$$
\bar{J}_{c}(\mathbf{w})=\mathrm{E}\left[J_{c}(\mathbf{w}, y(k))\right]
$$

with

$$
J_{c}(\mathbf{w}, y(k))=\rho \sum_{q=1}^{Q} \sum_{l=1}^{Q} \exp \left(-\frac{\left|y(k)-y_{q l}\right|^{2}}{2 \rho}\right) .
$$

In practice, the equaliser weights are adapted according to

$$
w_{i}(k+1)=w_{i}(k)+\mu \frac{\partial J_{c}(\mathbf{w}(k), y(k))}{\partial w_{i}}, \quad 0 \leq i \leq m-1 .
$$

Karaoguz and Ardalan [12] applied this blind equaliser to 4-QAM channels.

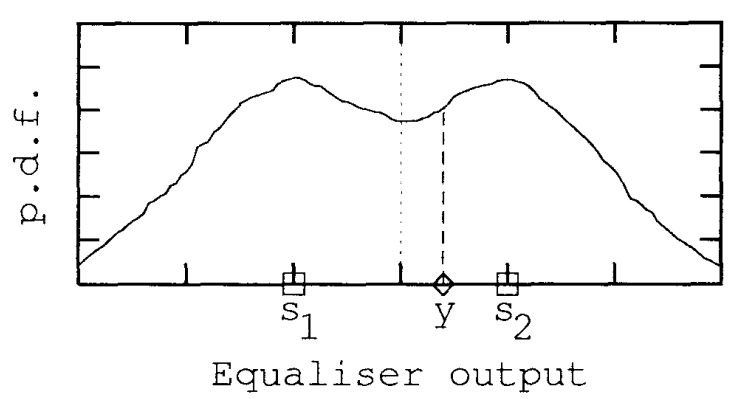

Fig. 1. Probability density function of a distorted 2-ary PAM signal before equalisation is achieved.

A direct application of this algorithm to high-order QAM signals has some serious drawbacks. This is not just because the complexity of the algorithm increases as $M$ increases. Consider the simplest case depicted in Fig.1, the case of a real channel and 2-ary pulse amplitude modulation (PAM) symbols. The gradient of $J_{c}$ in this case becomes

$$
\begin{aligned}
\frac{\partial J_{c}}{\partial w_{i}}= & \left(-\exp \left(-\frac{\left(y(k)-s_{1}\right)^{2}}{2 \rho}\right)\left(y(k)-s_{1}\right)-\right. \\
& \left.\exp \left(-\frac{\left(y(k)-s_{2}\right)^{2}}{2 \rho}\right)\left(y(k)-s_{2}\right)\right) r(k-i) .
\end{aligned}
$$

Referring to Fig.1, when the equaliser output $y$ is closer to $s_{2}$, the algorithm adjusts the weights in favour of $s_{2}$ as reflected in the second term of (11). But it is possible that the correct decision may actually be $s_{1}$, and the algorithm also adjusts the weights towards this decision as indicated by the first term in (11). It is clear that the algorithm is trying to find a compromise for the two conflicting factors. In this simplest case, it is relatively easy for the algorithm to achieve a correct equalisation. For high-order QAM signals, there are more conflicting factors to consider and convergence speed will generally be slow. This problem can also be viewed through the objective function (8). For a large $M, \bar{J}_{c}(\mathbf{w})$ can be highly nonconvex and, therefore, a gradient algorithm may have difficulties in finding a desired solution.

\section{The multi-stage Blind Clustering algorithm}

We propose a multi-stage blind clustering procedure for high-order QAM signal constellations, which overcomes the difficulties associated with high signal levels. This blind equalisation procedure is best illustrated by considering the 16-QAM case, where the overall equalisation objective is decomposed into the following two stages.

In the first stage, a 4-cluster model is adopted with the 4 cluster centres being $\tilde{s}_{11}=-2-j 2, \tilde{s}_{12}=-2+j 2$, $\tilde{s}_{21}=2-j 2$ and $\tilde{s}_{22}=2+j 2$. The equaliser weights are adjusted using this equivalent "4-QAM" model through the 
(a) After stage one, equaliser outputs are classified into 4 clusters. $\square$ indicate 4 cluster centres.

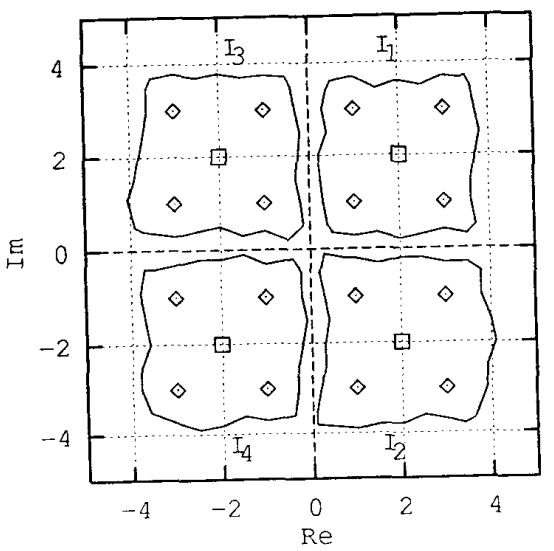

(b) After stage two, the eye pattern is opened. $\diamond$ indicate 16-QAM constellation.

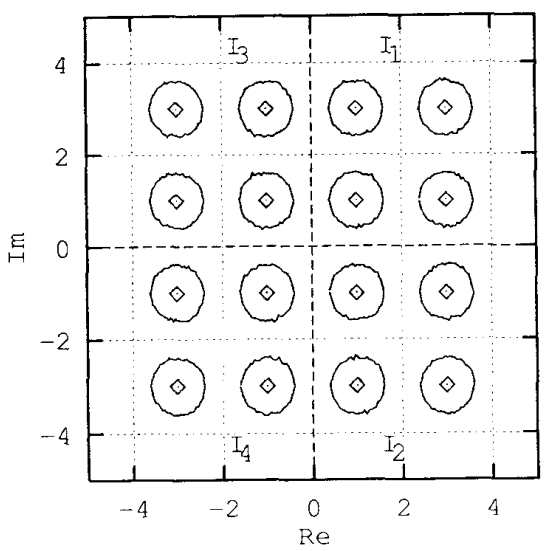

Fig. 2. Mlustration of two-stage blind clustering algorithm for 16 QAM equalisation.

gradient algorithm (10). The objective here is to achieve a roughly correct classification of equaliser outputs into the 4 quadrants $I_{i}, 1 \leq i \leq 4$, in the complex plane as illustrated in Fig.2 (a). At the second stage, the 16-cluster model is adopted with the 16 cluster means being $s_{q l}, 1 \leq q, l \leq 4$. This cluster model is divided into 4 sub-models, one for each $I_{i}$. If the equaliser output is in $I_{i}$, the corresponding 4-cluster sub-model is used to adapt the equaliser weights via the gradient algorithm (10). The aim of this stage is to reach the correct eye-open solution as illustrated in Fig.2 (b).

This clustering procedure is upwardly extendable. In general, the task of $M$-QAM equalisation, where $M=2^{2 L}$, can be achieved using an $L$-stage blind clustering equaliser. Because each stage can be accomplished easily, the overall convergence of the equaliser is achieved faster. A further advantage is that the complexity of the algorithm does not increase as $M$ increases, and is always comparable to the complexity of the 4-QAM case. Since the equaliser weights are adapted using a 4-cluster sub-model at each recursion via (10) this algorithm is only slightly more complex than the CMA.

A good rule for selecting the value of $\rho$ is that $\rho$ should be less than half of the distance between two adjacent cluster centres. If $\rho$ is too large, a desired separation among clusters may not be achieved. When a too small $\rho$ is used, the algorithm attemps to impose a tight control in the size of clusters and may fail to do so. Apart from these two extreme cases, the performance of the algorithm does not critically depend on the value of $\rho$. Intuition suggests that the blind clustering algorithm can achieve a desired equalisation in the face of insufficiently white input signals. A consequence of non-white input signals is that the equaliser outputs no longer form circularly distributed clusters around symbol points. However, as long as these clusters are sufficiently separated, the correct signal constellation is restored.

(a) three-stage blind clustering, 5000 samples used in adaptation.

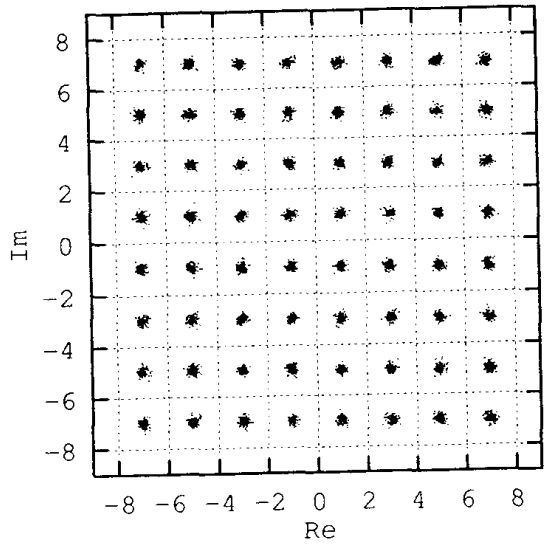

(b) CMA, 10000 samples used in adaptation.

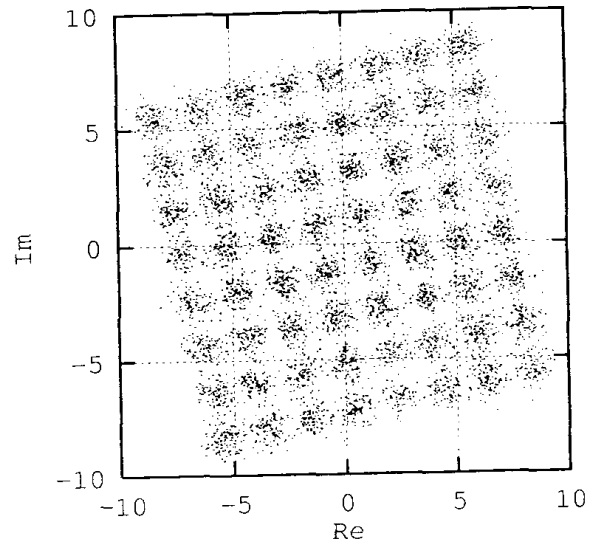

Fig. 3. Performance comparison for a 64-QAM 24-Mbits/s microwave fading channel. 


\begin{tabular}{|c|c|c|}
\hline Tap No. & Real & Imaginary \\
\hline 0 & 0.0145 & -0.0006 \\
1 & 0.0750 & 0.0176 \\
2 & 0.3951 & 0.0033 \\
3 & 0.7491 & -0.1718 \\
4 & 0.1951 & 0.0972 \\
5 & -0.2856 & 0.1896 \\
6 & 0.0575 & -0.2096 \\
7 & 0.0655 & 0.1139 \\
8 & -0.0825 & -0.0424 \\
9 & 0.0623 & 0.0085 \\
10 & -0.0438 & 0.0034 \\
11 & 0.0294 & -0.0049 \\
12 & -0.0181 & 0.0032 \\
13 & 0.0091 & 0.0003 \\
14 & -0.0038 & -0.0023 \\
15 & 0.0019 & 0.0027 \\
16 & -0.0018 & -0.0014 \\
17 & 0.0006 & 0.0003 \\
18 & 0.0005 & 0.0000 \\
19 & -0.0008 & -0.0001 \\
20 & 0.0000 & -0.0002 \\
21 & 0.0001 & 0.0006 \\
\hline
\end{tabular}

TABLE I

A TELEPHONE CHANNEL IMPULSE RESPONSE.

\section{PERFORMANCE COMPARISON}

The performance of the multi-stage blind clustering algorithm is compared with that of the CMA using two examples. The first example is a 64-QAM 24-Mbits/s microwave channel. The transmission pulse has a raisedcosine characteristics with a rolloff factor 0.5 . A three-path Rayleigh fading channel $v(t)=c_{0}(t) u(t)+c_{1}(t) u\left(t-\tau_{1}\right)+$ $c_{2}(t) u\left(t-\tau_{2}\right)$ is simulated with a fading rate $5 \mathrm{~Hz}$, where $u(t)$ is the transmitter output and $v(t)$ is the channel output; $\tau_{1} \approx T_{s} / 10, \tau_{2} \approx T_{s} / 3$ and $T_{s}$ is the symbol duration; and the root mean powers of the both real and imaginary components of $c_{i}(t)$ are $\left[\begin{array}{lll}0.7 & 0.6 & 0.5\end{array}\right]$. The signal to noise ratio (SNR) is $34 \mathrm{~dB}$. Receiver outputs are sampled at symbol rate and passed to an equaliser of 9 taps. The threestage blind clustering algorithm used 2000 samples for the stage one with $\rho=3.6,2000$ samples for the stage two with $\rho=1.8,1000$ samples for the stage three with $\rho=0.7$, and an adaptive gain $\mu=0.0003$ for all the three stages. After the above adaptation, the equaliser outputs are plotted in Fig.3 (a), where the stage-three adaptation was kept on. For the CMA, the adaptive gain had to be chosen as $\mu=0.1 \times 10^{-6}$ to avoid divergence. After an adaptation of 10000 samples, the equaliser outputs obtained with continuous adaptation are shown in Fig.3 (b).

The second example is a telephone channel listed in Table I. A non-white 16-QAM symbol sequence is generated as follows. A pseudorandom binary sequence of a repetive period 256 is generated, and every four bits are converted into a 16-QAM symbol. This is designed to sim- ulate the case reported in [4]. The SNR was $24 \mathrm{~dB}$ and the equaliser had 23 taps. For the CMA, the adaptive gain was $\mu=0.00001$. The equaliser outputs after the adaptation of 20000 samples are shown in Fig.4 (a), where it is seen that the CMA converged to a false solution. For the two-stage blind clustering algorithm, an adaptive gain of $\mu=0.002$ were employed for the both stages, $1500 \mathrm{sam}$ ples were used in the stage one with a $\rho=1.6$, and 2000 samples in the stage two with a $\rho=0.6$. After the adaptation, the equaliser outputs are plotted in Fig.4 (b). The different sizes of the clusters in Fig.4 (b) was due to nonequiprobable 16-QAM symbol points. As expected, the distributions of the clusters were not circular. However, the correct signal constellation was restored.

(a) CMA, 20000 samples used in adaptation.

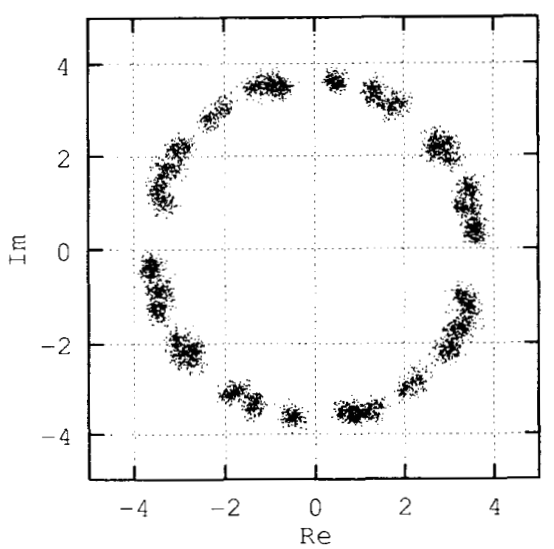

(b) two-stage blind clustering, 3500 samples used in adaptation.

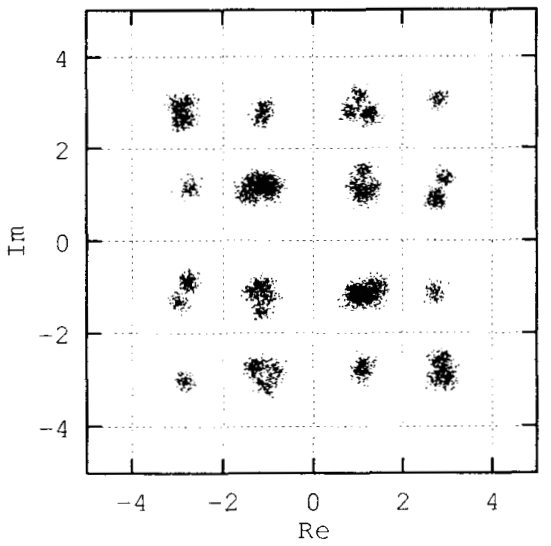

Fig. 4. Performance comparison for a 16-QAM telephone channel with non-white input symbol sequence.

\section{CONCLUSIONS}

A novel multi-stage blind clustering algorithm has been presented for equalisation of high-order QAM channels. It has been shown that the multi-stage blind clustering algo- 
rithm offers significant improvement in performance over the CMA at the cost of slightly increased computational complexity. This new blind adaptive algorithm has been shown to be very robust in the presence of non-white input symbol sequence.

\section{REFERENCES}

[1] Y. Sato, "A method of self-recovering equalization for multilevel amplitude-modulation systems," IEEE Trans. Communications, Vol.COM-23, pp.679-682, 1975.

[2] D. Godard, "Self-recovering equalization and carrier tracking in two-dimensional data communication systems," IEEE Trans. Communications, Vol.COM-28, pp.1867-1875, 1980.

[3] J.R. Treichler and B.G. Agee, "A new approach to multipath correction of constant modulus signals," IEEE Trans. Acoustics, Speech and Signal Processing, Vol.ASSP-31, No.2, pp.459-472, 1983.

[4] J.R. Treichler, "Application of blind equalization techniques to voiceband and RF modems," in Preprints 4th IFAC Int. Symposium Adaptive Systems in Control and Signal Processing (France), 1992, pp.705-713.

[5] N.K. Jablon, "Joint blind equalization, carrier recovery, and timing recovery for high-order QAM signal constellations," IEEE Trans. Signal Processing, Vol.40, No.6, pp.1383-1398, 1992.

[6] G.B. Giannakis and J.M. Mendel, "Identification of nonminimum phase system using higher order statistics," IEEE Trans. Acoustics, Speech and Signal Processing, Vol.ASSP-37, pp.360-377, 1989.

[7] H.-H. Chiang and C.L. Nikias, "Adaptive deconvolution and identification of nonminimum phase FIR systems based on cumulants," IEEE Trans. Automatic Control, Vol.AC-35, pp.36-47, 1990.

[8] F.-C. Zheng, S. McLaughlin and B. Mulgrew, "Blind equalization of nonminimum phase channels: higher order cumulant based algorithm," IEEE Trans. Signal Processing, Vol.41, No.2, pp.681691, 1993.

[9] N. Seshadri, "Joint data and channel estimation using blind trellis search techniques," IEEE Trans. Communications, Vol.42, No.2/3/4, pp.1000-1011, 1994.

[10] K. Giridhar, J.J. Shynk and R.A. Itis, "A modified Bayesian algorithm with decision feedback for blind adaptive equalization," in Preprints 4th IFAC Int. Symposium Adaptive Systems in Control and Signal Processing (France), 1992, pp.737-742.

[11] E. Zervas, J. Proakis and V. Eyuboglu, "A quantized channel approach to blind equalization," in Proc. ICC'92 (Chicago), 1992, Vol.3, pp.351.8.1-351.8.5.

[12] J. Karaoguz and S.H. Ardalan, "A soft decision-directed blind equalization algorithm applied to equalization of mobile communication channels," in Proc. ICC'92 (Chicago), 1992, Vol.3, pp.343.4.1-343.4.5. 